\title{
Síntese e atividade biológica do derivado 6-formil-oxamniquina
}

\author{
Adélia Emília de Almeida ${ }^{*}$, Ana Luiza Ribeiro de Souza ${ }^{2}$, Silmara Marques Allegretti ${ }^{3}$, Tarsila \\ Ferraz Frezza ${ }^{3}$, Mara Cristina Pinto ${ }^{4}$, Antônio Gilberto Ferreira ${ }^{5}$, Man Chin Chung1 \\ ${ }^{1}$ Laboratório de Pesquisa e Desenvolvimento de Fármacos, Departamento de Fármacos e \\ Medicamentos, Faculdade de Ciências Farmacêuticas, Universidade Estadual Paulista "Júlio de Mesquita Filho", \\ ${ }^{2}$ Departamento de Fármacos e Medicamentos, Faculdade Faculdade de Ciências \\ Farmacêuticas, Universidade Estadual Paulista "Júlio de Mesquita Filho", \\ ${ }^{3}$ Laboratório de Helmintologia, Departamento de Parasitologia, Instituto de Biologia, \\ Universidade Estadual de Campinas, \\ ${ }^{4}$ Laboratório de Parasitologia, Departamento de Ciências Biológicas, Faculdade de \\ Ciências Farmacêuticas, Universidade Estadual Paulista "Júlio de Mesquita Filho", \\ ${ }^{5}$ Departamento de Química Orgânica, Universidade Federal de São Carlos
}

*Correspondência:

A.E. Almeida

Faculdade de Ciências Farmacêuticas - UNESP

Rod. Araraquara-Jaú, km. 01

14801-902 - Araraquara - SP, Brasil

E-mail: almeidaa@fcfar.unesp.br
A esquistossomose, uma importante doença no Brasil, é causada por trematódeo pertencente ao gênero Schistosoma, atingindo milhões de pessoas, numa das maiores regiões endêmicas dessa doença em todo globo. O principal objetivo desse trabalho foi sintetizar o derivado 6-formil-oxamniquina e avaliar sua atividade biológica. O derivado 6-formil-oxamniquina foi obtido por oxidação da oxamniquina com dióxido de manganês empregando diclorometano como solvente, à temperatura ambiente, por 24 horas. Sua obtenção foi confirmada por espectrometria na região de infravermelho e espectroscopia de $R M N{ }^{13} \mathrm{C}$ e ${ }^{1} \mathrm{H}$, apresentando atividade similar quando comparada à oxamniquina comercial (Mansil $\left.{ }^{\circledR}\right)$.

\section{INTRODUÇÃO}

A esquistossomose, também chamada esquistossomíase ou bilharziose, é uma doença produzida por trematódeos do gênero Schistosoma. Entre as 19 espécies reconhecidas, cinco infectam primariamente o homem: $S$. mansoni, S. haematobium, S. intercalatum, S. japonicum e $S$. mekongi (Organização Mundial de Saúde, 1994; Shekhar, 1991; World Health Organization, 2006).

Essa doença originou-se na África Central, disseminando-se para o norte desse continente, com as correntes migratórias ao longo do vale do Nilo. Sua transferência para o Brasil teve origem no primeiro grande ciclo econômico, as chamadas monoculturas de exportação, que neces- sitavam de grandes contingentes de mão-de-obra com o mais baixo custo possível. Iniciava-se a escravatura no Brasil com a comercialização de escravos trazidos de países africanos, para a região Nordeste brasileira. Esses grandes contingentes populacionais migrantes encontraram precárias condições de vida e de trabalho, em diferentes regiões e áreas, favorecendo a disseminação da enfermidade. A existência de meio ambiente físico propício, com presença do hospedeiro intermediário, e ineficazes programas de controle permitiram que a esquistossomose se tornasse endêmica (Amaral, Porto, 1994; Carvalho et al., 1998).

No Brasil, é popularmente conhecida como "barrigad'água" ou "mal do caramujo", atingindo milhões de pessoas, numa das maiores regiões endêmicas dessa doença em todo 
globo (Rey, 2002). Acredita-se que cerca de 200 milhões de pessoas estejam infectadas no mundo todo e 600 milhões estejam sob o risco de contrair a doença (Almeida et al., 2006; CDC, 2006; World Health Organization, 2006).

Apenas dois fármacos estão disponíveis no país para o tratamento dessa enfermidade: oxamniquina e praziquantel. Os efeitos adversos provocados pelo praziquantel caracterizam-se por tonturas, náuseas, cefaléias, dores abdominais, sonolência e exantema urticariforme. O S. mansoni é altamente suscetível à oxamniquina, entretanto é considerada o fármaco de $2^{\mathrm{a}}$ escolha devido aos seus efeitos colaterais, principalmente àqueles ligados ao $\mathrm{SNC}$. Estes efeitos, associados à grande incidência da parasitose em nosso país e, ao risco do aparecimento de resistência, sempre presente, demonstram a grande importância do desenvolvimento de novos agentes esquistossomicidas (Barberato-Filho et al., 2002; CDC, 2006; El-Hamouly et al., 1988; Parise-Filho et al., 2004).

O mecanismo de ação da oxamniquina ainda não está completamente elucidado. Alguns autores sugerem que a oxamniquina é enzimaticamente convertida através de uma quinase ou sulfotransferase para um éster, o qual pode alquilar o DNA do Schistosoma (Cioli et al., 1993), sendo, portanto o grupo meltilhidroxil (presente na posição 6) essencial à atividade biológica (Figura 1).

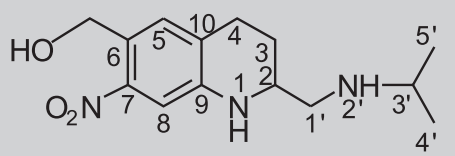

FIGURA 1 - Estrutura química da oxamniquina (6-hidroximetil-2-isopropilaminometil-7-nitro-1,2,3,4tetraidroquinolina).

No que se refere à introdução de novos fármacos, os processos de modificação molecular (Bundgaard, 1991; Burger, 1995; Chung, Ferreira, 1999) são os mais promissores.

O presente trabalho teve como objetivo realizar a modificação no grupo meltilhidroxila presente na posição 6 visando à obtenção do derivado 6-formil-oxamniquina e avaliar a sua atividade biológica.

\section{MATERIAL E MÉTODOS}

\section{Material}

Todos os solventes e reagentes utilizados foram de grau analítico e a oxamniquina foi gentilmente doada pelo Centro de Pesquisa da Pfizer.

\section{Equipamentos}

Os dados de absorção na região de infravermelho foram obtidos em comprimento de onda de $4000-400 \mathrm{~cm}^{-1}$ em pastilhas de $\mathrm{KBr}$, utilizando Espectrofotômetro Shimadzu FTIR-8300, à temperatura ambiente.

Os espectros de RMN ${ }^{13} \mathrm{C},{ }^{1} \mathrm{H}$ foram obtidos em Espectrômetro BRÜKER DRX400 (9,4 Tesla) com tetrametilsilano como padrão interno e DMSO-d ${ }_{6}$ como solvente, à temperatura constante de $25^{\circ} \mathrm{C}$. Os deslocamentos químicos foram registrados em $\mathrm{d}$ (ppm).

As faixas de fusão foram determinadas em aparelhos de ponto de fusão capilar MEL-TEMP II.

\section{Síntese do derivado 6-formil-oxamniquina}

Em balão de fundo redondo foram solubilizados $3,6 \mathrm{mmol}(1,0 \mathrm{~g})$ oxamniquina em $100,0 \mathrm{~mL}$ de diclorometano. Em seguida, foram colocados $34,5 \mathrm{mmol}$ $(3,0 \mathrm{~g})$ de dióxido de manganês divididos em 2 porções e adicionadas com 15 minutos de intervalo. O meio reacional foi mantido à temperatura ambiente, sob agitação por 24 horas. Após filtração, o solvente foi evaporado e o precipitado obtido foi seco. A reação é apresentada no Esquema 1, esta foi acompanhada por CCD utilizando-se como eluente clorofórmio/metanol/ácido acético 95:5:3 (v:v:v), empregando como revelador solução alcoólica de 2,4-dinitrofenilidrazina, revelador específico para aldeído.

\section{Avaliação da atividade biológica}

O ensaio foi realizado no Laboratório de Helmintologia do Departamento de Parasitologia UNICAMP. Foram utilizados camundongos Swiss fêmeas

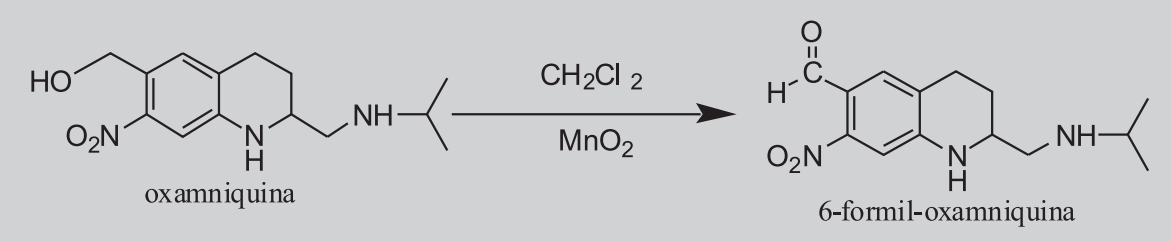

ESQUEMA 1 - Síntese do derivado 6-formil-oxamniquina. 
SPF com 30 dias de idade. Os animais foram infectados, com 70 cercárias do Schistosoma mansoni, da linhagem $\mathrm{BH}$, por imersão caudal durante 2 horas com exposição à luz e calor de acordo com o método descrito por Olivier e Stirewalt (1952). Após 45 dias de infecção, os animais, então apresentando ovos viáveis nas fezes, foram tratados. A dose selecionada foi de $100 \mathrm{mg} / \mathrm{kg}$ de peso. Esta dose representa a ED99 da oxamniquina e tem sido empregada freqüentemente nos ensaios de atividade biológica. O veículo utilizado foi água destilada. Os camundongos foram distribuídos em 3 grupos:

a) Cinco (5) animais infectados receberam oxamniquina comercial (OXAc) (Mansil ${ }^{\circledR}$, Pfizer), na concentração de $100 \mathrm{mg} / \mathrm{kg}$, administrada por tubagem gástrica, em dose única $0,3 \mathrm{~mL}$.

b) Cinco (5) animais infectados receberam o derivado 6-formil-oxamniquina (OXAf), na concentração de $100 \mathrm{mg} / \mathrm{kg}$, administrada por tubagem gástrica, em dose única $0,3 \mathrm{~mL}$.

c) Cinco (5) animais infectados receberam solução salina $\mathrm{NaCl} 0,9 \%$ por tubagem gástrica, em dose única $0,3 \mathrm{~mL}$ - grupo controle.

Todos os animais dos 3 grupos foram eutanaziados por deslocamento cervical, quinze dias após o tratamento. Os vermes foram coletados por perfusão do sistema portahepático (Yolles et al., 1947) e de cada camundongo foram retirados fragmentos do intestino delgado para realizar o método do oograma, para identificação dos estágios de desenvolvimento dos ovos. O fígado, intestino, baço e pulmões foram retirados e comprimidos entre duas placas de vidro para a visualização dos granulomas. As fezes dos camundongos também foram analisadas, utilizando-se o método quantitativo de Kato, modificado por Katz, Pellegrino e Memoria (1972), visando à contagem de ovos.

Todos os procedimentos acima relatados foram também realizados para os grupos de animais controle, ou seja, aqueles que receberam somente a solução de $\mathrm{NaCl}$.

Os dados foram transformados em logaritmos e analisados estatisticamente por análise de variância e comparação múltipla de médias pelo teste de Tukey.

O protocolo experimental foi conduzido de acordo com os princípios éticos do Colégio Brasileiro de Experimentação Animal (COBEA) - protocolo n ${ }^{\circ}$ 1117-1.

\section{RESULTADOS E DISCUSSÃO}

A obtenção do derivado desejado foi confirmada pelos dados de faixa de fusão, IV e RMN ${ }^{13} \mathrm{C}$. A faixa de fusão da oxamniquina encontrada na literatura é $147-149^{\circ} \mathrm{C}$ (Merck, 2006), enquanto que o derivado 6-formiloxamniquina apresentou $75-83,2^{\circ} \mathrm{C}$.
As bandas de absorção observadas no IV $\left(\mathrm{KBr}, \mathrm{cm}^{-1}\right)$ que contribuem para a caracterização da oxamniquina são: $3328\left(v_{\mathrm{N}-\mathrm{H}}\right.$ e $v_{\mathrm{O}-\mathrm{H}}$ livre $), 2972\left(\mathrm{v}_{\mathrm{C}-\mathrm{H}}\right.$ aromático $), 2900-2846$ $\left(\mathrm{v}_{\mathrm{C}-\mathrm{H}}, \mathrm{v}_{\mathrm{CH} 2} \mathrm{e} \mathrm{v}_{\mathrm{CH} 3}\right.$ simétrico e assimétrico $), 1620\left(\mathrm{v}_{\mathrm{C}=\mathrm{C}}\right.$ aromático), $1517\left(\mathrm{v}_{\mathrm{NO} 2}\right), 1332\left(\mathrm{v}_{\mathrm{NO} 2}\right.$ simétrico $), 1292\left(\mathrm{v}_{\mathrm{C}-\mathrm{O}}\right), 1051$ $\left(v_{\mathrm{O}-\mathrm{H}}\right.$ álcool primário e $\left(\mathrm{v}_{\mathrm{C}-\mathrm{N}}\right)$. Enquanto no derivado 6-formiloxamniquina foi observada a presença em: $3352\left(v_{\mathrm{N}-\mathrm{H}}\right), 2964$ $\left(\mathrm{v}_{\mathrm{CH}-\mathrm{CH} 3}\right.$ simétrica e assimétrica), $3846\left(\mathrm{v}_{\mathrm{C}-\mathrm{H}}\right.$ aldeído conjugado em efeito orto), 1666 ( $\mathrm{v}_{\mathrm{C}=\mathrm{O}}$ aldeído conjugado ligação hidrogênio intramolecular), $1596\left(v_{\mathrm{C}=\mathrm{C}}\right.$ anel aromático $), 1517\left(\mathrm{v}_{\mathrm{NO} 2}\right.$ assimétrico), $1336\left(\mathrm{v}_{\mathrm{NO} 2}\right.$ simétrico) e em $1051\left(\delta_{\mathrm{C}-\mathrm{O}}\right)$.

$\mathrm{Na}$ análise por $\mathrm{RMN}{ }^{13} \mathrm{C}$ do derivado 6 -formiloxamniquina foi observado um pico em 186,3 ppm, correspondendo à presença de aldeído. A comprovação de obtenção é verificada pela análise $\mathrm{RMN}{ }^{1} \mathrm{H}$ em que observase o deslocamento químico do hidrogênio aldeídico- $\mathrm{COH}$ em 9,82 ppm. Como ele está se correlacionando com os deslocamentos químicos do anel aromático, com isso fica demonstrado que o radical aldeídico está de fato ligado ao anel e, portanto a oxidação de álcool para aldeído funcionou, ou seja, foi obtido o derivado 6-formil oxamniquina (Tabela I).

Resultados obtidos com a linhagem $\mathrm{BH}$ de S. mansoni indicaram que com 45 dias de infecção, a quantidade de vermes encontrados nos camundongos foi menor no grupo tratado com (OXAc) (5 machos, 7 fêmeas, 6 casais), que no grupo tratado com o (OXAf) ( 9 machos, 12 fêmeas, 35 casais), porém essa diferença não foi estatisticamente significativa ( $p>0,05)$. Ambos os tratamentos diferiram estatisticamente do controle $(p<0,01)$ que apresentou maior quantidade de vermes (46 machos, 49 fêmeas e 42 casais. Quanto ao número total de vermes houve diferença estatística entre os três tratamentos $(\mathrm{p}<0,01)$, sendo que a oxamniquina e o seu derivado não diferiram entre si, mas em ambos, menos vermes foram encontrados em relação ao controle.

No oograma encontramos maior quantidade de ovos imaturos de $1^{\circ}, 2^{\circ}, 3^{\circ}$ e $4^{\circ}$ estágios de desenvolvimento nos animais tratados com o (OXAf) (Tabela II), mas em contrapartida na contagem de ovos nas fezes, o tratamento com o (OXAf) reduziu a oviposição sendo encontrados 686 ovos. $\mathrm{g}^{-1}$ e 1100 ovos. $\mathrm{g}^{-1}$ para os tratados com (OXAc) (Tabela III). O grupo controle apresentou 2384,5 ovos. $\mathrm{g}^{-1}$ sendo que esse número corresponde ao dobro de ovos encontrados em OXAc e ao triplo dos ovos liberados após tratamento com OXAf. Em relação ao $5^{\circ}$ estágio de desenvolvimento, ou seja, ovos maduros, o grupo tratado com o (OXAf), (OXAc) e controle apresentaram 88, 72 e 61 ovos, respectivamente. A análise de variância apresentou diferença entre os três tratamentos $(\mathrm{p}=0,02)$. O controle não diferiu da (OXAc) $(p>0,05)$ nem do seu derivado $(p>0,05)$, porém em seu derivado (OXAf) mais ovos foram encontrados em relação à $(\mathrm{OXAc})(\mathrm{p}<0,05)$. 
Tabela I - Deslocamento químico '(ppm) e constante de acoplamento $\mathrm{J}(\mathrm{Hz})$ para o derivado 6-formil oxamniquina em DMSO-d6

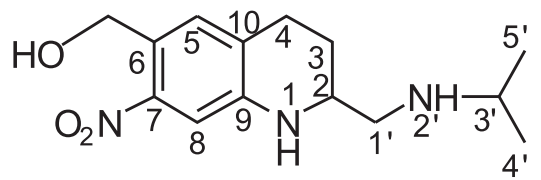

\begin{tabular}{|c|c|c|c|}
\hline No. & ${ }^{\prime}{ }^{1} \mathrm{H}$ & ${ }^{\prime 13} \mathrm{C}$ & $\mathrm{gHMBC}$ \\
\hline 2 & $3,34-3,45 \mathrm{~m}$ & 50,9 & 51,5 e 24,9 \\
\hline 3 & $1,45-1,62 \mathrm{~m} 1,85-1,97 \mathrm{~m}$ & 23,8 & 123,$4 ; 50,9 ; 48,2$ e 24,9 \\
\hline 4 & $2,69-2,79 \mathrm{~m}$ & 24,9 & 150,$1 ; 130,8$ e 123,4 \\
\hline 5 & $7,49 \mathrm{~s}$ & 130,8 & 186,$3 ; 150,1 ; 123,4 ; 115,5 ; 107,0$ e 24,9 \\
\hline 6 & $-\ldots$ & 115,5 & ---- \\
\hline 7 & $-\ldots$ & 150,1 & 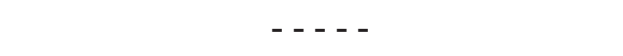 \\
\hline 8 & $7,10 \mathrm{~s}$ & 107,0 & 186,$3 ; 150,1 ; 123,4 ; 115,5$ e 24,9 \\
\hline 9 & ---- & 150,2 & ---- \\
\hline 10 & $-\ldots$ & 123,4 & $--_{-}^{-}$ \\
\hline 1 ' & $2,48-2,59 \mathrm{~m} 2,59-2,72 \mathrm{~m}$ & 51,5 & 24,9 e 23,8 \\
\hline 3' & $2,69-2,79 \mathrm{~m}$ & 48,2 & 150,$2 ; 130,8 ; 123,4 ; 50,9 ; 23,7$ e 22,9 \\
\hline $4^{\prime}, 5^{\prime}$ & $1,01 \mathrm{~d} J=6,57$ & 22,9 & 48,2 \\
\hline$>\mathrm{COH}$ & $9,82 \mathrm{~s}$ & 186,3 & 186,$3 ; 150,1 ; 130,8 ; 123,4$ e 115,5 \\
\hline
\end{tabular}

$\mathrm{s}$ - singleto; $\mathrm{d}$-dubleto; $\mathrm{m}$ - multipleto.

TABELA II - Oograma (total de ovos nos diferentes estágios encontrados para cada tratamento)

\begin{tabular}{lccc}
\hline Estágio do Ovo & $\mathrm{NaCl} 0,9 \%$ & OXAc & OXAf \\
\hline $1^{\text {o }}$ & 4 & 1 & 37 \\
$2^{\text {o }}$ & 13 & 3 & 19 \\
$3^{\text {o }}$ & 17 & 1 & 56 \\
$4^{\text {o }}$ & 3 & 3 & 3 \\
$5^{\text {o }}$ & 61 & 72 & 88 \\
mortos & 12 & 19 & 12 \\
Total de ovos & $110(\mathrm{a})$ & $99(\mathrm{ab})$ & $215(\mathrm{ac})$ \\
encontrados* & & &
\end{tabular}

*As mesmas letras não apresentam diferenças significativas na análise estatística $\mathrm{p}>0,05$.

TABELA III - Número de ovos de S. mansoni por grama de fezes de camundongos infectados e tratados com OXAc e OXAf (exame de fezes: Kato Katz)

\begin{tabular}{lc}
\hline Tratamento & Ovos/grama de fezes \\
\hline $\mathrm{NaCl} 0,9 \%$ & 2384,5 \\
OXAc & 1100 \\
OXAf & 686 \\
\hline
\end{tabular}

Em relação à formação de granulomas no fígado, intestino, baço e pulmões todos os grupos apresentaram granulomas em grande quantidade $(+++)$ no fígado. Nos grupos tratados com (OXAc) e com seu derivado tiveram formação menor de granulomas no intestino, baço e pulmões $(+)$ (Tabela IV).

Em números absolutos, o tratamento com a (OXAc) apresentou resultado levemente superior em relação ao seu derivado quanto ao número total de vermes recuperados no intestino, ou seja, houve maior mortandade, porém como estatisticamente não foi detectada diferença pode-se considerar que os dois apresentaram similaridade quanto a esse parâmetro Em relação ao oograma podemos inferir que há interrupção na oviposição feita pelas fêmeas que permaneceram vivas após o tratamento, mas que se restabeleceu depois. Observamos este fato pela quantidade de ovos imaturos existentes que é maior para o grupo tratado com o (OXAf) em relação à (OXAc). Entretanto, verificamos quantidade significativamente menor de ovos eliminados nas fezes para este mesmo grupo em relação ao tratado com (OXAc). Como há maior quantidade de ovos imaturos no oograma, mas, também, menor quantidade de ovos eliminados nas fezes, podemos supor que houve interrupção na oviposição e que se restabeleceu depois. Pelos parâmetros analisados os dois tratamentos apresentam perfil similar. 
TABELA IV - Resultados da contagem de granulomas e presença de vermes de $S$. mansoni nos órgãos dos camundongos, excetuando as veias mesentéricas

\begin{tabular}{lcccc}
\hline Tratamento & Fígado & Intestino & Baço & Pulmões \\
\hline NaCl 0,9\% & +++ & + & ++ & ++ \\
& 2 vermes & & & \\
OXAc & +++ & limpo & + & + \\
& 2 vermes & & & \\
OXAf & +++ & limpo & + & + \\
\hline
\end{tabular}

+++ Órgão com muitos granulomas; ++ órgão com quantidade intermediária de granulomas e + órgão com poucos granulomas.

\section{CONCLUSÕES}

O derivado 6-formil-oxamniquina foi obtido e a substituição do grupo meltilhidroxil pela função aldeído não alterou a sua atividade. Pretende-se, em uma próxima etapa, obter novos derivados na tentativa de elucidar a importância do substituinte $\mathrm{OH}$ (hidroxila) no mecanismo de ação, uma vez que o derivado aldeídico obtido apresentou equivalente atividade biológica.

\section{ABSTRACT \\ Synthesis and biological activity of 6-formyl- oxamniquine derivative}

Schistosomiasis, an important disease in Brazil, is caused by a trematode of the genus Schistosoma, reaching millions of person in one of the most endemic region of this disease in the whole globe. The main goal of this work was to syntetize the 6-formyl-oxamniquine derivative and evaluate its biological activity. The 6-formyl-oxamniquine derivative was obtained by the oxidation of oxamniquine with $\mathrm{MnO}$, applying $\mathrm{CH}_{2} \mathrm{Cl}_{2}$ as solvent at room temperature for 24 hours. The obtaintion of 6-formyl-oxamniquine derivative compound was confirmed by IR spectroscopy and ${ }^{13} C N M R$ and ${ }^{1} H N M R$, presenting similar activity when compared to the commercial oxamniquine (Mansil ${ }^{\mathbb{}}$ ).

UNITERMS: Schistosomiasis. Ooxamniquine. Schistosoma mansoni.

\section{AGRADECIMENTOS}

Os autores agradecem a colaboração do Centro de Pesquisa da Pfizer, localizado em Sandwich (Reino Unido), pela doação da oxamniquina utilizada nesse estudo.

\section{REFERÊNCIAS BIBLIOGRÁFICAS}

ALMEIDA, A. E.; FERREIRA, A. G.; CRESPI, M. S.; ANDRADE, Z. A.; CHUNG, M. C. Synthesis and thermal study of prodrug of oxamniquine. J. Therm. Anal. Calorim., v.83, n.2, p.277-281, 2006.

AMARAL, R. S.; PORTO, M. A. S. Evolução e situação do controle da esquistossomose no Brasil. Rev. Soc. Bras. Med. Trop., v.27, n.2, p.73-90, 1994.

BARBERATO-FILHO, S.; ARGIONI, C.; PINTO, C. P. L. S.; CHIODELLI, S. G.; VELLOSA, S. A. G.; SILVA, R. M.; SILVEIRA, M.A. B. Synthesis and evaluation of new oxamniquine derivatives. Int. J. Pharm., v.233, n.1-2, p.35-41, 2002.

BUNDGAARD, H. Novel chemical approaches in prodrug design. Drugs Fut., v.16, n.5, p.443-58, 1991.

BURGER, A. The conceptual background and development of medicinal chemistry. In: WOLFF, M. E. (Ed.), Burger's Medicinal Chemistry and Drug Discovery: Principles and Practice, John Wiley \& Sons: New York, 1995. v.1, p.1-8

CARVALHO, E. M.; ACIOLI, M. D.; BRANCO, M. A. F.; COSTA, A. M.; CESSE, E. A. P.; ANDRADE, A. G.; MELLO, E. M. L. L. Evolução da esquistossomose na Zona da Mata Sul de Pernambuco. Epidemiologia e situação atual: controle ou descontrole? Cad. Saúde Pública, v.14, n.4, p.787-795, 1998.

CDC. Health and Safety Topics, Atlanta, G.A.: U.S. Department of health and Human Services, CDC, 2007. Disponível em: <http://www.cdc.gov/default.htm.>. Acesso em: 20 de out. 2007.

CHUNG, M. C.; FERREIRA, E. I. O processo de latenciação no planejamento de fármacos. Quim. Nova, v.22, n.1, p.75-84, 1999.

CIOLI, D.; PICA-MATTOCCIA, L.; ARCHER, S. Drug resistance in schistosomes. Parasitol. Today, v.9, n.5, p.162-166, 1993.

EL-HAMOULY, W.; PICA-MATTOCIA, L.; CIOLI, D.; SCHWARTZ, H. M.; ARCHER, S. Studies on some derivatives of oxamniquine. J. Med. Chem., v.31, n.8, p.1629-1631, 1988. 
KATZ, N.; PELLEGRINO, J.; MEMORIA, J. M. P. Quantitative oogram method in Cebus monkeys experimentally infected with Schistosoma mansoni. $J$. Parasitol., v.52, n.5, p.917-919, 1972.

MERCK index. 14.ed. Whitehouse Station, 2006. p.1192.

OLIVIER, L.; STIREWALT, M. A. An efficient meted for exposure of mice to cercarie of Schistosoma mansoni. J. Parasitol., v.38, p.19-23, 1952.

ORGANIZAÇÃO MUNDIAL DE SAÚDE, 1994. O Controle da Esquistossomose: Segundo Relatório do Comitê de Especialistas da OMS. Rio de Janeiro: Editora Fiocruz. 110p. (Technical Reports Series, 830).

PARISE-FILHO, R.; ARAÚJO,A.A. S.; SANTOS-FILHO, M.; MATOS, J. R.; SILVEIRA, M. A. B.; BRANDT, C. A. Thermoanalytical characterization of potentially schistosomicide polymeric derivatives. J. Therm. Anal. Calorim., v.75, n. 2, p.487-494, 2004.
REY, L. Esquistossomíase Mansônica In: Bases da Parasitologia Médica. $3^{\mathrm{a}}$ ed. Rio de Janeiro: Guanabara Koogan, 2002, p.413-443.

SHEKHAR, K. C. Schistosomiasis drug therapy and treatment consideration. Drugs, v.42, n.3, p.379-405, 1991.

WORLD HEALTH ORGANIZATION. WHO, 2006. Schistosomiasis and intestinal parasites control. Planning and technical guidance. Communicable diseases prevention and control. Disponível em: $<\mathrm{http}$ :/ www.WHO.Ch./cds.>. Acesso em: 16 de out. 2006.

YOLLES, T. K.; MOORE, P. V.; DE GINSTI, D. L.; RIPSON, C. A.; MELENEY, H. E. A technique for the perfusion of laboratory animals for the recovery of schistosomes. J. Parasitol., v.33, p.419-426, 1947.

Recebido para publicação em 21 de fevereiro de 2008 Aceito para publicação em 21 de agosto de 2008 